\title{
COMPARATIVE STUDY ON NEURAL OSCILLATION IN THE PROCEREBRUM OF THE TERRESTRIAL SLUGS INCILARIA BILINEATA AND LIMAX MARGINATUS
}

\author{
SHIGENORI KAWAHARA*, SHOICHI TODA, YASUYOSHI SUZUKI, SATOSHI WATANABE \\ AND YUTAKA KIRINO \\ Laboratory of Neurobiophysics, School of Pharmaceutical Sciences, The University of Tokyo, 7-3-1 Hongo, \\ Bunkyo-ku, Tokyo 113, Japan \\ Accepted 21 April 1997
}

\begin{abstract}
Summary
Coherent oscillatory activities in procerebral neurones have been described in Limax maximus; however, the electrical properties of the procerebrum of other terrestrial molluscs are less well understood. We have examined oscillatory activity in the procerebrum of Incilaria bilineata and Limax marginatus. The local field potential measured in the procerebrum of I. bilineata showed repetitive peaks which had the opposite polarity from those measured in $L$. marginatus. Optical measurement of membrane potential using a potential-sensitive dye, di-4-ANEPPS, showed that

slow and a rapid component in both species. The rapid component was most pronounced in the internal mass of $I$. bilineata but was prominent in the cell mass of $L$. marginatus. The superior tentacle nerve, which projects to the terminal mass, also showed oscillations in synchrony with those of the procerebrum. These results suggest that oscillations in procerebral interneurones are commonly generated in a region adjacent to the terminal mass and that these oscillations may affect the membrane potential of the neurones constituting the superior tentacle nerve.
\end{abstract} the oscillations in I. bilineata occurred mainly in the internal mass while those in $L$. marginatus were located in the cell mass. An analysis of the waveform revealed that the depolarizing phase of the oscillations consists of both a
Key words: slug, Incilaria bilineata, Limax marginatus, olfaction, neural oscillation, potential-sensitive dye, optical recording.

\section{Introduction}

Terrestrial molluscs can learn to recognize the odour of food (Croll and Chase, 1980; Sahley et al. 1981; Yamada et al. 1992; Teyke, 1995). This learning includes the recognition of sensory information based on the memory they have and, accordingly, requires highly developed machinery for the active processing of sensory information. Metabolic analysis using 2-deoxyglucose autoradiography (Chase, 1985) revealed that olfactory information is processed in the procerebrum, an area of the brain that is well-developed in terrestrial molluscs. The structure of the procerebrum in snails and slugs has been documented (Zs-Nagy and Sakharov, 1970; Chase and Tolloczko, 1989). Although the morphology of the procerebrum differs among mollusc species, there are basic similarities in that the procerebrum is constructed largely from the somatic region, called the cell mass, and the neuropile region, which is subdivided into a terminal mass and an internal mass. The neuropile region receives projections from the superior and inferior tentacle nerves as well as having a cerebropedal connection (Chase and Tolloczko, 1989; Gelperin et al. 1993), which means that interactions between procerebral neurones and input and output pathways occur mainly in the neuropile region. Studies on the procerebrum of the slug Limax maximus using electrophysiological techniques (Gelperin and Tank, 1990; Gelperin et al. 1993) and optical techniques with a potential-sensitive dye (Delaney et al. 1994; Kleinfeld et al. 1994) revealed that the membrane potential of procerebral neurones oscillates coherently with phase delays along the distal-to-proximal axis of the cell mass. Furthermore, these oscillations are modulated by an odour stimulus to the superior tentacular nose (Gelperin and Tank, 1990; Delaney et al. 1994) or to the inferior tentacular nose (Kimura et al. 1993). It is of interest to determine whether the oscillatory activity of procerebral neurones is a general phenomenon in terrestrial molluscs and also whether there are differences in the electrical properties of the somatic and neuropile regions. An understanding of the mechanisms involved in the generation of oscillations in terrestrial slugs will aid in determining the role of these oscillations in olfactory processing and learning. We report here our findings in the procerebrum of two terrestrial slugs, Incilaria bilineata and Limax marginatus.

Materials and methods

Specimens of the slugs Incilaria bilineata (Benson) and

*e-mail: kawahara@oldwiseman.f.u-tokyo.ac.jp. 
Limax marginatus (Müller) were collected in the open field or were bred from eggs in our laboratory (Kawahara et al. 1994). I. bilineata weighing 2-5 g and $L$. marginatus weighing $1-2 \mathrm{~g}$ were used. I. bilineata was anaesthetized by cooling in crushed ice for $30 \mathrm{~min}$, whereas L. marginatus was anaesthetized by injecting $0.2-0.5 \mathrm{ml}$ of isotonic $\mathrm{Mg}^{2+}$ solution into the body cavity. The cerebral ganglion was isolated and placed in a chamber filled with saline of the following composition (in $\mathrm{mmoll}^{-1}$ ) for I. bilineata (the value given in parentheses is that for L. marginatus): $\mathrm{NaCl}, 30.8$ (70.0); $\mathrm{KCl}, 1.5$ (2.0); $\mathrm{CaCl}_{2}$, 4.0 (4.9); $\mathrm{MgCl}_{2}, 3.1$ (4.7); glucose, 5.0 (5.0); Hepes, 5.0 (5.0); $\mathrm{pH} 7.6$ buffered with $\mathrm{NaOH}$. The isotonic $\mathrm{Mg}^{2+}$ solution used to anaesthetize L. marginatus had the following composition (in $\mathrm{mmoll}^{-1}$ ): $\mathrm{MgCl}_{2}$, 57.6; glucose, 5.0; Hepes, 5.0; $\mathrm{pH} 7.6$. The composition of saline for I. bilineata was based on an analysis of the inorganic ion composition and the osmolarity of the haemolymph, and resembled that for Incilaria fruhstorferi (Kawahara et al. 1994). The saline used for $L$. marginatus was also based on an analysis of its haemolymph (personal communication from Dr Tetsuya Kimura).

For morphological analysis of the procerebrum, the superior tentacle nerve was back-filled with $\mathrm{NiCl}_{2}$. The cut end of the tentacle nerve was drawn into a glass pipette filled with $10 \%$ $\mathrm{NiCl}_{2}$ and left there for $7 \mathrm{~h}$ at room temperature $\left(22-25^{\circ} \mathrm{C}\right)$. The preparation was then washed with saline, and the stained neurones were visualized by adding a few drops of a saturated solution of rubeanic acid in ethanol. Connective tissue surrounding the cerebral ganglion was softened by enzymatic treatment [I. bilineata: $1 \%$ protease type IV (Sigma) for $30 \mathrm{~min}$; L. marginatus: $0.2 \%$ actinase E (Kaken Pharmaceutical Co., Ltd, Chiba, Japan) for $20 \mathrm{~min}$ ] at $20^{\circ} \mathrm{C}$, stained with $0.1 \%$ Trypan Blue (Wako Pure Chemical Industries, Ltd, Osaka, Japan) for $10 \mathrm{~min}$, and then carefully removed with fine forceps. Trypan Blue stained the connective tissue, thus making its removal easier.

To stain procerebral interneurones, the lipophilic fluorescent dyes 1, 1'-dioctadecyl-3,3,3',3'-tetramethylindocarbocyanine perchlorate and 3,3'-dioctadecyloxacarbocyanine perchlorate (DiI and DiO; Molecular Probes, Inc., Oregon, USA) were used. After removing the connective tissue surrounding the cerebral ganglion, as described above, the preparations were left in $10 \%$ neutral formalin buffer solution (Wako Pure Chemical Industries, Ltd, Osaka, Japan) for 1-2 days. Cell bodies of the procerebral neurones were stained with a saturated solution of $\mathrm{DiI}$ or $\mathrm{DiO}$ in ethanol from the tip of a glass pipette (tip diameter $20-100 \mu \mathrm{m}$ ). After washing with formalin, the stained preparation was stored in a dark box for 1 week to permit the dye to diffuse along the lipid membranes of the neurones, and the preparation was then sectioned $(10-15 \mu \mathrm{m})$ in a cryostat. To stain the tract of the superior tentacle nerve running into the procerebrum, the cut end of the tentacle nerve was stained with DiI.

Extracellular field potentials were recorded from the surface of the procerebrum using a glass electrode (tip diameter $20-50 \mu \mathrm{m})$ filled with saline and from the cut end of the superior tentacle nerve using a glass suction electrode. These field potentials were bandpass-filtered between 0.5 and $30 \mathrm{~Hz}$. For optical measurement using a potential-sensitive dye, the cerebral ganglion of $I$. bilineata was desheathed as described above, while the cerebral ganglion of $L$. marginatus was left intact. The ganglia were stained with a saturated solution (approximately $0.02 \% \mathrm{w} / \mathrm{v}$ ) of di-4-ANEPPS (Molecular Probes, Inc., Oregon, USA) in saline for $20 \mathrm{~min}$ at $20^{\circ} \mathrm{C}$, washed with saline, and placed on a glass coverslip which constituted the bottom of a chamber filled with saline. These preparations were left for at least $1 \mathrm{~h}$ before the start of optical measurements in order to allow the dye to diffuse into the cerebral ganglion. The orientation of the cerebral ganglion was determined so that the mixing of optical signals from the somatic and the neuropile regions of the procerebrum was minimized (same orientation as shown in Fig. 3), and a mesh of nylon strings was used for fixation. The cerebral ganglion of L. marginatus was further immobilized with $4 \%$ lowmelting-point agarose (Sigma). The preparation was mounted on an inverted fluorescence microscope (IX70: Olympus) equipped with a $\times 10$ objective lens (NA 0.40), a bandpass excitation filter $(530-550 \mathrm{~nm})$, a dichroic mirror $(565 \mathrm{~nm})$ and a long-pass emission filter $(580 \mathrm{~nm})$. A $100 \mathrm{~W}$ halogen lamp with a d.c. power supply was used as the light source. Optical signals were recorded with the $128 \times 128$ photodiode array system (HR Deltaron 1700: Fuji Photo Film), attached to the bottom TV port of the microscope. To generate an image, the optical signals were accumulated for $38.4 \mathrm{~ms}$.

Using mathematical software (PV-WAVE Personal Edition, Visual Numerics Inc., Texas, USA) running on an IBM-compatible computer, each image was rebinned to form one constructed from $64 \times 64$ pixels. Each pixel covered a $13 \mu \mathrm{m} \times 13 \mu \mathrm{m}$ area of the specimen. The change in the fluorescence intensity of each pixel reflected the change of field potential in that area, i.e. the mean membrane potential in the field of the pixel. Because di-4-ANEPPS stained the neuropile more strongly than the somatic region, the fluorescence changes were normalized by calculating the fractional change in fluorescence intensity $(\Delta F / F)$ as follows:

$$
\Delta F / F=\left[F(x, y, t)-F_{\mathrm{av}}(x, y, t)\right] / F_{\mathrm{av}}(x, y, t),
$$

where $F(x, y, t)$ is the fluorescence intensity of a pixel located at $(x, y)$ at a given time $(t)$, and $F_{\mathrm{av}}(x, y, t)$ is a time-averaged fluorescence intensity corrected for photobleaching and calculated as follows. First, the mean fluorescence intensities between the depolarizing peaks of oscillation were calculated. This cycle-averaged fluorescence intensity decreased as a result of photobleaching by $0.1-0.9 \%$ during the period of optical measurement, typically $40 \mathrm{~s}$. The value of $F_{\text {av }}(x, y, t)$ was then calculated as a regression line fitted to a series of cycle-averaged fluorescence intensities $(r=0.98-1.00)$.

The $\Delta F / F$ values represent a depolarization or a hyperpolarization relative to the time-averaged field potential in each pixel. In Fig. 6 , the $\Delta F / F$ values are coded as pseudocolours. Pixels not focused on the specimen were excluded from the calculation and are coloured black. In Fig. 7, repetitive cycles of oscillation were accumulated and averaged 
after matching the depolarization peaks. Analysis was also made using a Macintosh computer and a public-domain NIH Image program (developed at the US National Institutes of Health and available from the Internet by anonymous File Transfer Protocol from zippy.nimh.nih.gov or on floppy disk from the National Technical Information Service, Springfield, Virginia, USA, part number PB95-500195GEI).

\section{Results}

Morphology of the procerebra of Incilaria bilineata and Limax marginatus

The descriptive terms used to describe the orientation of the cerebral ganglion of Incilaria bilineata follow those used for Limax maximus (Cooke and Gelperin, 1988; Kleinfeld et al. 1994). Thus, the 'posterior view' of the cerebral ganglion corresponds morphologically to the 'dorsal view' of the snail Achatina fulica (Chase and Tolloczko, 1989).

The morphology of the procerebrum of I. bilineata differed from that of L. marginatus (Figs 1A, 2A). To visualize the arrangement of the superior tentacle nerve and the procerebrum, the superior tentacle nerve was stained by backfilling with $\mathrm{NiCl}_{2}$. The superior tentacle nerve of I. bilineata projected to the procerebrum at its distal end (Fig. 1A), while that of L. marginatus projected at the proximal end (Fig. 2A). The procerebrum of both species can be divided into three regions; the cell mass, the terminal mass and the internal mass.
Tracts of the tentacle nerve of I. bilineata ran along the anterior and posterior surfaces of the terminal mass from the distal to the proximal end. Those of L. marginatus ran along the anterior and posterior surfaces of the internal mass, and another tract projected into the terminal mass. The direction of the projection of the tentacle nerve to the terminal mass was from the proximal to the distal end. The tract projecting to the terminal mass of $I$. bilineata overlapped with tracts running over the surface of the terminal mass and could not be seen from the outside.

The orientation of the procerebrum in the cerebral ganglion of I. bilineata also differed from that of L. marginatus. The internal mass of $I$. bilineata appeared as a small lump on the antero-dorsal surface of the cerebral ganglion, while that of $L$. marginatus was seen on the anterior face. Thus, in the anterior view of L. marginatus (Fig. 2A), the cell mass underlies the terminal mass and the internal mass. To distinguish clearly between the neurite region and the somatic region, the procerebrum of L. marginatus should be viewed from the dorsal side, whereas that of I. bilineata has to be viewed from the anterior side (see also Fig. 3C,D).

The projections of procerebral neurones were investigated using a lipophilic fluorescent dye, DiI. When a large area of the cell mass of the desheathed procerebrum was marked with DiI, the neurites of the procerebral cells were shown to extend to the internal mass of the procerebrum (Figs 1B, 2B). The internal mass could be distinguished from the terminal mass by the

Fig. 1. Morphology of the procerebrum of Incilaria bilineata. The dorsal side is towards the top of the picture. (A) Whole-mount anterior view of the left hemisphere of the cerebral ganglion. The tentacle nerve was stained by backfilling with $\mathrm{NiCl}_{2}$ and visualized with rubeanic acid. The stained area appears darker than surrounding tissues. (B-D) Fluorescent photomicrographs of the sectioned procerebra (frontal sections) stained with DiI, which appears as a white colour in these figures. (B) Projection of the procerebral neurones. DiI was applied to the cell bodies. (C) Projection of the tentacle nerve in the procerebrum. DiI was applied to the cut end of the tentacle nerve. (D) Fibre projections to the terminal mass. DiI was applied within the terminal mass by inserting a
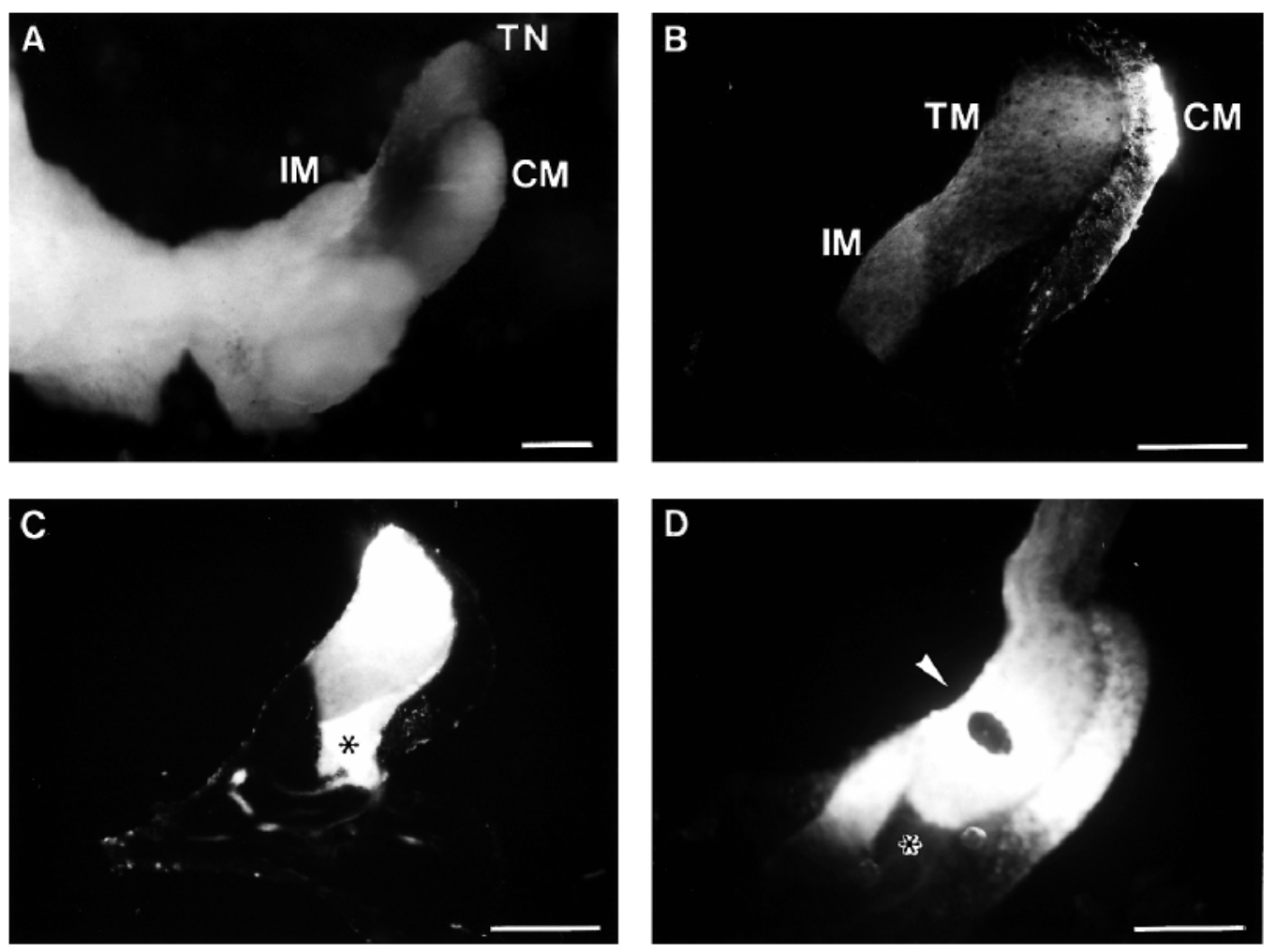
glass pipette filled with the dye at the location shown by the arrowhead. The asterisk in $\mathrm{C}$ indicates one of the regions stained when DiI was applied to the cut end of the superior tentacle nerve. The asterisk in D indicates that the same region was not stained when DiI was applied to the terminal mass. TN, superior tentacle nerve; CM, cell mass; TM, terminal mass; IM, internal mass. Scale bars, $200 \mu \mathrm{m}$. 

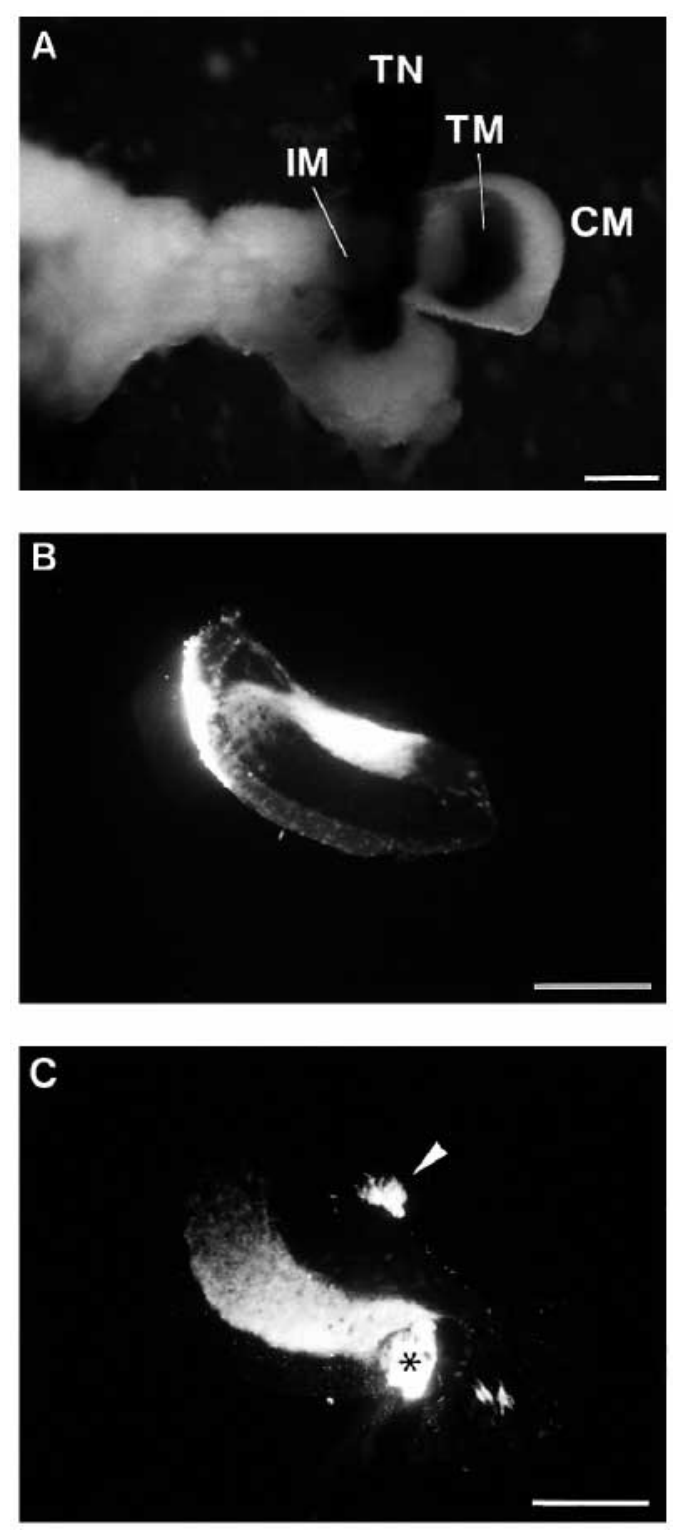

Fig. 2. Morphology of the procerebrum of Limax marginatus. (A) Whole-mount anterior view of the left hemisphere of the cerebral ganglion. The dorsal side is towards the top of the figure. The tentacle nerve was stained by back-filling with $\mathrm{NiCl}_{2}$. (B,C) Fluorescent photomicrographs of the sectioned procerebra (horizontal sections) showing the projection of the procerebral neurones (B) and the projection of the tentacle nerve $(\mathrm{C})$. The anterior side is towards the top of the figure. DiI was applied to the cell bodies (B) or to the cut end of the tentacle nerve (C). The arrowhead in $\mathrm{C}$ indicates tracts of the tentacle nerve running over the anterior surface of the internal mass; the asterisk marks the posterior surface of the internal mass. $\mathrm{TN}$, superior tentacle nerve; CM, cell mass; TM, terminal mass; IM, internal mass. Scale bars, $200 \mu \mathrm{m}$.

intensity of the fluorescence. Marking the internal mass with DiI resulted in staining of the terminal and cell masses, but not the tentacle nerve (data not shown). The projection of the superior tentacle nerve to the procerebrum was also investigated using DiI. When DiI was applied to the cut end of the superior tentacle nerve, the tentacle nerve of $I$. bilineata was observed to project into the terminal mass from the distal to the proximal end, and proceeded deeper into the cerebral ganglion (region labelled with an asterisk), while projections to the internal mass were not observed (Fig. 1C). Because the tract of the tentacle nerve might possibly pass through the terminal mass and extend outside the procerebrum (region labelled with an asterisk in Fig. 1C), termination of the tract within the terminal mass was confirmed using retrograde staining of the tentacle nerve by applying DiI to the terminal mass (Fig. 1D). This treatment resulted in staining of the tentacle nerve, the internal mass and the cell mass. The tract of the tentacle nerve outside the procerebrum was not stained (region labelled with an asterisk in Fig. 1D), indicating that the tract of the tentacle nerve projecting to the terminal mass terminates there. In L. marginatus, part of the tentacle nerve ran over the anterior (arrowhead in Fig. 2C) and posterior surface (asterisk in Fig. 2C) of the internal mass, while the other part of the tentacle nerve projected to the terminal mass, but not to the internal mass (Fig. 2C).

The projection patterns of procerebral neurones in the neurite region were also visualized with lipophilic fluorescent dyes. When DiI was applied to the distal and proximal portions of the cell mass of I. bilineata with a space between them, both distal and proximal areas of the internal mass were stained; the distal staining could be traced back to the distal portion of the cell mass and the proximal staining to the proximal portion (Fig. 3A). When DiI was applied only to the distal portion or only to the proximal portion of the cell mass, only the distal or the proximal portion of the internal mass was stained (data not shown). Fig. 3B shows the procerebrum of L. marginatus stained with DiI and DiO. In this preparation, the distal portion of the cell mass was marked with $\mathrm{DiO}$ and the proximal portion with DiI. Thus, the distal portion of the internal mass was stained with $\mathrm{DiO}$ and the proximal portion was stained with DiI. These results indicate that the topology of the arrangement of the cell mass is preserved in the internal mass in both species.

\section{Oscillatory activity of the procerebrum of Incilaria bilineata and Limax marginatus}

The local field potentials of somatic and neuropile regions of the procerebrum were investigated using surface electrodes placed on the anterior side of the procerebrum. The preparation consisted of the left and right procerebra with the remaining cerebral ganglion attached. The other circumoesophageal ganglia and the buccal ganglion were removed. The local field potential of the cell mass was recorded from the edge of the procerebrum, an area where cell bodies of the procerebral neurones were visible under a dissecting microscope. The local field potential of the neuropile region was recorded from the internal mass, which formed a small lump and could be distinguished from the terminal mass.

In I. bilineata, the internal mass clearly showed repetitive activity, while the cell mass showed only a weak signal (Fig. 4A). The peak of the local field potential in the internal mass was positive and the amplitude varied with time. In 
Fig. 3. Projection pattern of the procerebral interneurones. (A) Whole-mount anterior view of the right procerebrum of $I$. bilineata. DiI was applied to the distal (small arrowhead) and to the proximal (large arrowhead) portions of the cell mass. This resulted in staining of distal (small arrow) and proximal (large arrow) portions of the internal mass. (B) Whole-mount dorsal view of the right procerebrum of L. marginatus. DiI was applied to the distal and $\mathrm{DiO}$ to the proximal portion of the cell mass. DiI appears orange and $\mathrm{DiO}$ green under the fluorescence microscope. Scale bars, $200 \mu \mathrm{m}$.

(C) Schematic drawing of the procerebrum of I. bilineata, showing the orientation of A. (D) Schematic drawing of the procerebrum of L. marginatus, showing the orientation of $\mathrm{B}$. TN, superior tentacle nerve; CM, cell mass; TM, terminal mass; IM, internal mass.
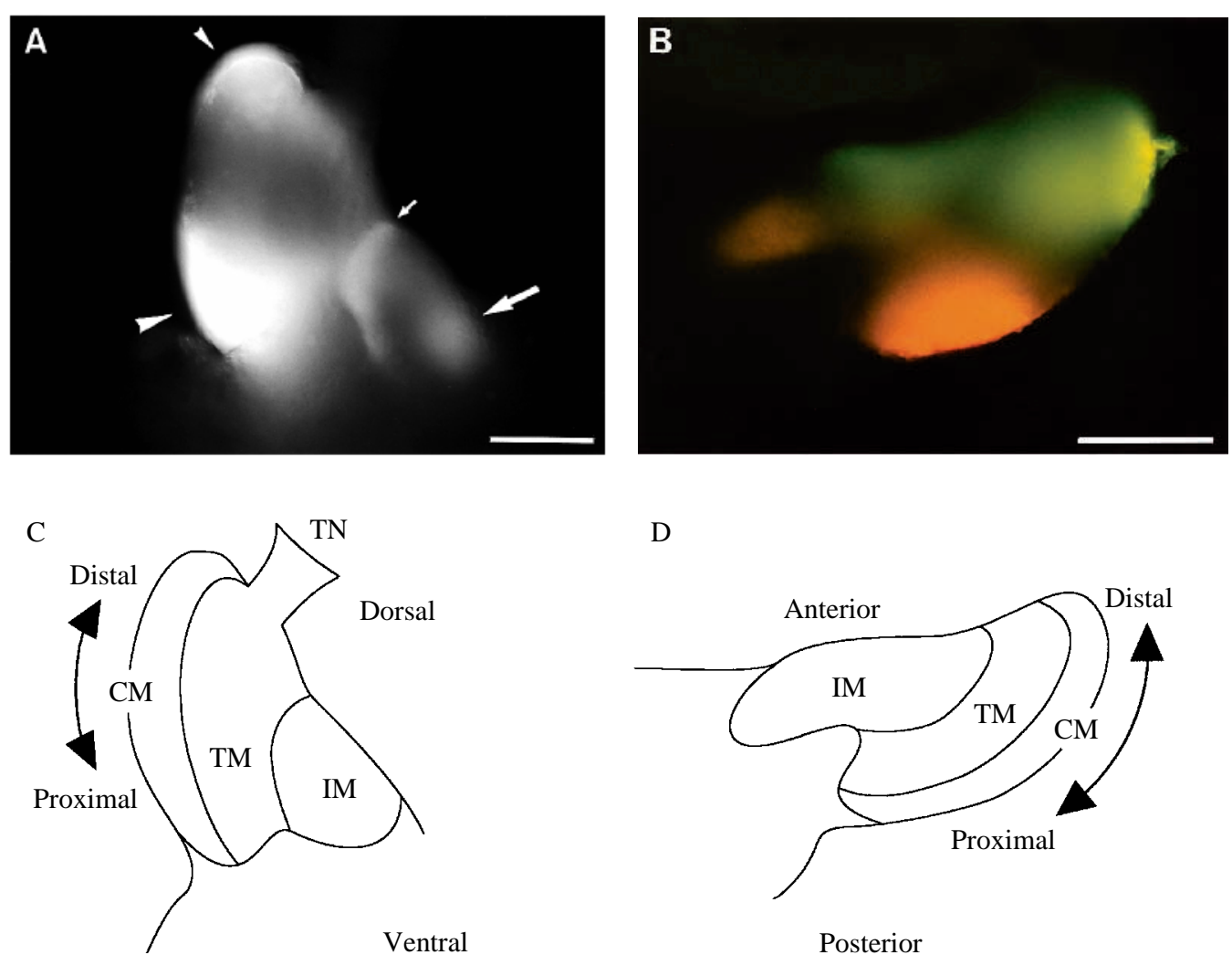

$\mathrm{D}$

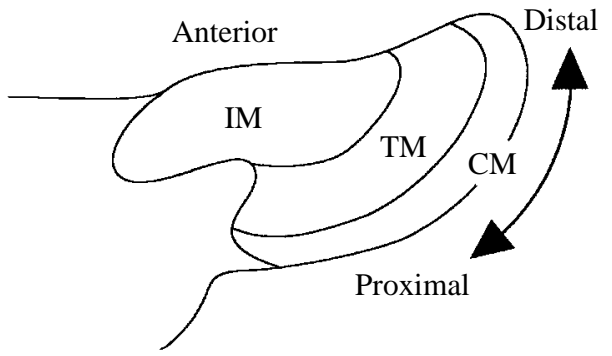

Posterior contrast, both the cell mass and the internal mass of $L$. marginatus showed regular oscillatory activity (Fig. 4B). The peaks of the local field potential in the cell mass were positive and in the internal mass they were negative, which was the opposite polarity to that of I. bilineata. The histogram in Fig. 5 shows the distribution of the frequency of the repetitive activity recorded in a single preparation. The frequency was defined as the inverse of the interval between the positive peaks. The histogram shows that the frequency of oscillation in a single preparation of $I$. bilineata was variable, whereas in L. marginatus it was constant. The mean frequency differed among preparations, ranging from 0.35 to $0.69 \mathrm{~Hz}$ in $I$. bilineata $(0.53 \pm 0.08 \mathrm{~Hz}$, mean \pm S.D.; $N=15)$ and from 0.55 to $1.00 \mathrm{~Hz}$ in L. marginatus $(0.77 \pm 0.15 \mathrm{~Hz}$, mean \pm S.D.; $N=24)$.

To investigate the spatio-temporal pattern of the oscillatory activity in the procerebrum of I. bilineata and L. marginatus in more detail, optical recordings using a potential-sensitive dye, di-4-ANEPPS, were made. Oscillations of fluorescence
A

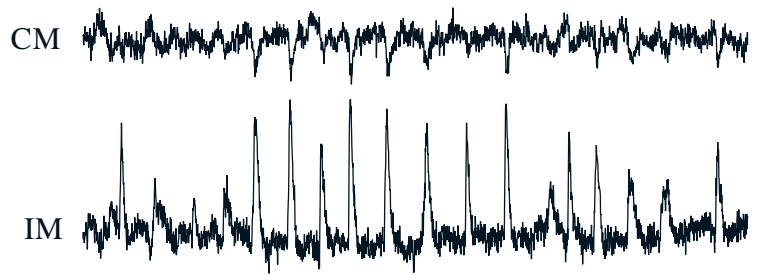

B
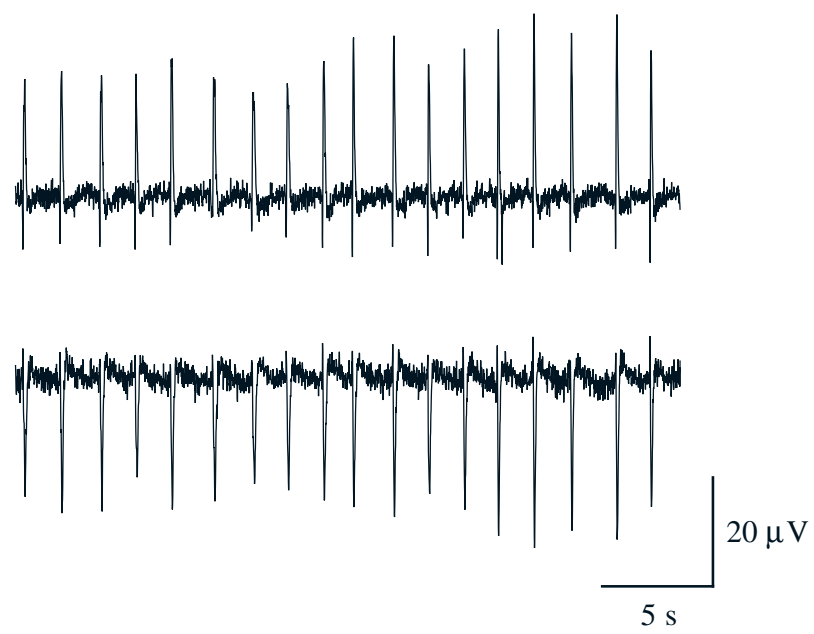

Fig. 4. Oscillation of the local field potential of the procerebrum of I. bilineata (A) and L. marginatus (B). Recordings were obtained extracellularly from the surface of the procerebrum in the isolated cerebral ganglion, using glass electrodes filled with saline (see Materials and methods). CM, cell mass; IM, internal mass. 


\section{S. KAWAHARA AND OTHERS}

intensity were observed in the procerebrum of both species of slugs. The mean frequency of oscillation in I. bilineata was $0.33 \pm 0.10 \mathrm{~Hz}$ (mean \pm S.D.; $N=12$ ) and that of $L$. marginatus was $0.70 \pm 0.12 \mathrm{~Hz}$ (mean \pm S.D.; $N=21$ ). The oscillation measured optically in I. bilineata had a tendency to be slower than that measured electrically in the intact cerebral ganglion, although no significant differences were detected by a twotailed Student's $t$-test $(P=0.15)$.

Oscillations were evident in the procerebrum of both species, but the spatio-temporal patterns differed in accordance with the differences in polarity of local field potential peaks. Fig. 6 shows successive images during a little over one cycle of oscillation in the procerebrum of both species. In I. bilineata (Fig. 6A), depolarization was initiated at the distal end of the internal mass (frame numbers 2 and 56), which is the projection site of distal procerebral interneurones (see Fig. 3), and moved towards the proximal end of the internal mass (frame numbers 3-13 and 57-67), where the neurites of proximal procerebral interneurones project. However, in $L$. marginatus, oscillations with the largest amplitude were in the cell mass (Fig. 6B). Depolarization was initiated at the distal end of the procerebrum (frame numbers 7 and 48) and moved along the somatic layer towards the proximal end (frame numbers 8-22 and 49-63).

To increase the signal-to-noise ratio of the weakly stained regions and to identify the region with an oscillation in phase with that of the procerebral interneurones, several sets of successive images constituting each cycle of oscillation were accumulated and averaged after matching the depolarization peaks (Fig. 7). In accordance with the images in Fig. 6, the amplitude of the oscillation in the internal mass of I. bilineata (trace 4 in Fig. 7A) was greater than that in the other masses, while the amplitude of the oscillation in the cell mass of $L$. marginatus (trace 2 in Fig. 7B) was greater than that in the neurite regions. Comparing the waveform of the oscillation in each mass, the depolarizing phase of the oscillation in the internal mass of $I$. bilineata and in the cell mass of $L$. marginatus consisted of an early slow component and a later rapid component, while the wave in the other masses consisted largely of a slow component. To highlight the region which contained the rapid component, the distribution of the width of the depolarization peak at half-height is shown in Fig. 8. Pixels with a maximum $\Delta F / F$ of less than $5 \%$ of that of the procerebrum were considered to have little coherent oscillation and hence were excluded from calculation of the peak width. The map of the peak width shows that, in I. bilineata (Fig. 8A), the peak width in the internal mass was smaller than in the other masses, whereas in L. marginatus (Fig. 8B) the peak width was smaller in the cell mass, indicating that these regions contained more of the rapid component than the other regions.

In addition to oscillations in the procerebrum, the superior tentacle nerve also showed oscillatory activity in synchrony with the procerebral neurones in both species of slugs (trace 1 in Fig. 7A,B), whereas in other regions outside the procerebrum there were no oscillations (traces 5, 6 and 7 in Fig. 7A,B). This tentacular nerve oscillation was further confirmed by extracellular recording of the field potential of the superior tentacle nerve, using a suction electrode (Fig. 9). The field potential of the superior tentacle nerve clearly showed oscillatory activity in synchrony with the oscillations of the local field potential of the procerebrum. Furthermore, in I. bilineata, the amplitude of oscillations in the tentacle nerve varied with time and correlated well with changes in the amplitude of oscillations measured in the internal mass (Fig. 9A).

From optical recordings of the irregular oscillatory pattern of I. bilineata, the amplitude of the rapid component of depolarization, which was most prominent in the internal mass, varied with time (Fig. 10). The degree of hyperpolarization in the procerebrum after the depolarization peak depended on the amplitude of the rapid component, and the superior tentacle nerve also became intensely hyperpolarized after rapid depolarization of the internal mass. Because the slope of the slow component of depolarization was little changed in spontaneous oscillations, the interval between the peaks depended on the degree of hyperpolarization just after the depolarization peak and hence on the amplitude of the rapid component of the preceding depolarization peak. Fig. 11 shows scatter diagrams of the peak interval against the size of the rapid component of the preceding depolarization peak. The size of the rapid component of a given peak was defined as the total of the $\Delta F / F$ values above the threshold, determined manually as a border-line between slow and rapid components (see the broken line in Fig. 10). The graphs show a positive
Fig. 5. Distribution of oscillation frequencies in a single preparation. Frequencies were defined as the inverse of the interval between the positive peaks of the local field potential measured in the internal mass of I. bilineata (A) or in the cell mass of L. marginatus (B). Histograms were constructed from data recorded for $4 \mathrm{~min}$.

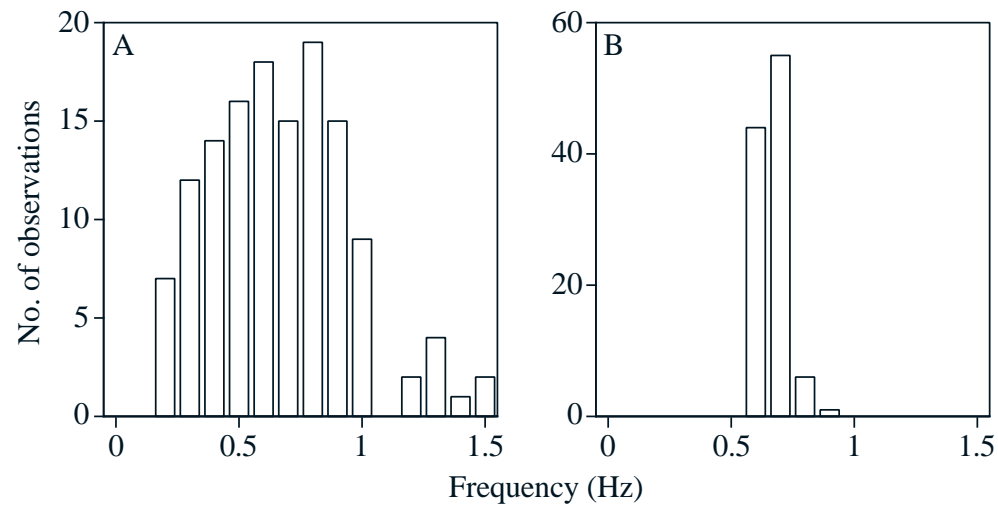


A

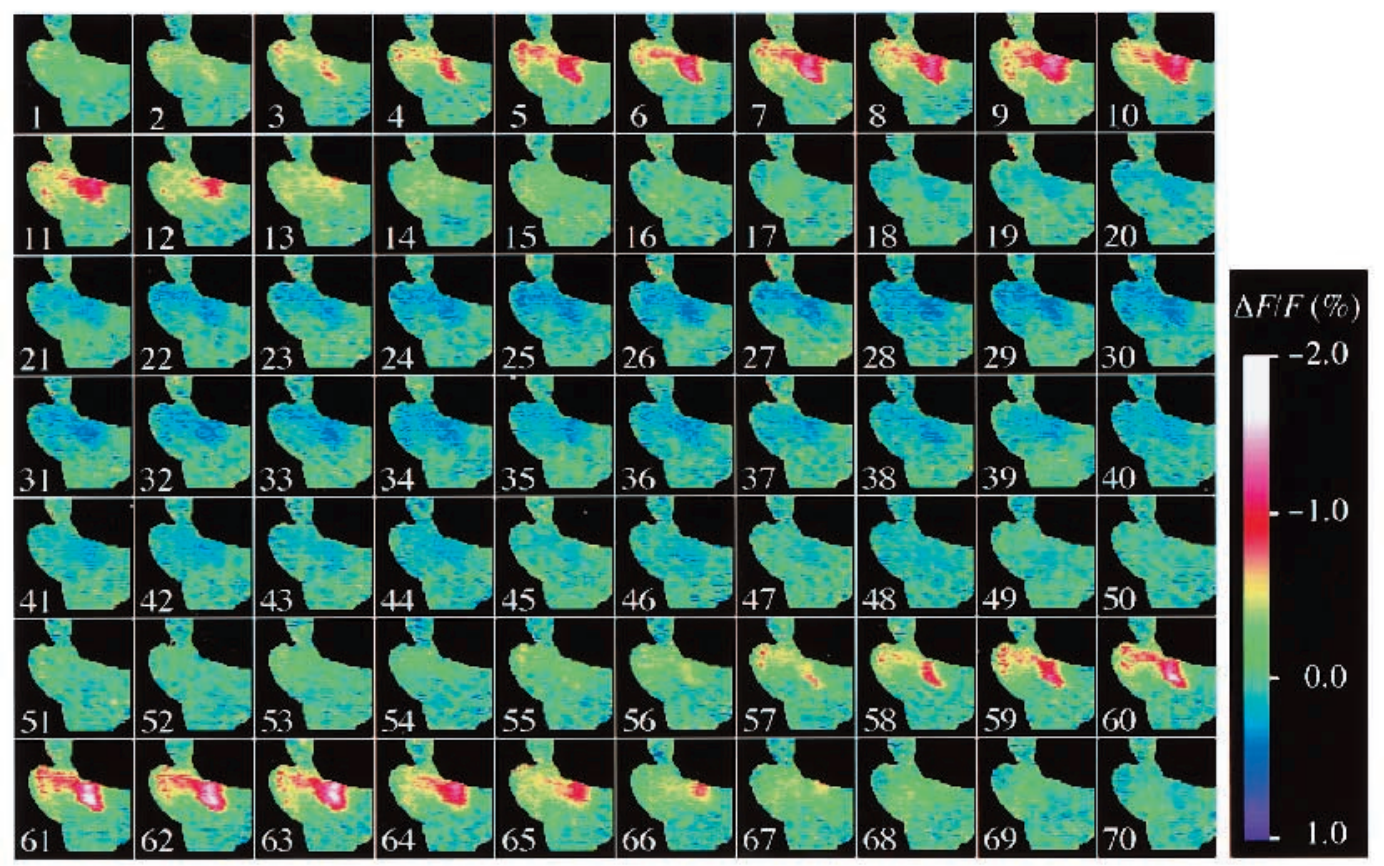

B

Fig. 6. Spatio-temporal pattern of the oscillation of the field potential in the procerebrum measured with the potentialsensitive dye di-4-ANEPPS. (A) I. bilineata (anterior view). (B) L. marginatus (dorsal view). The orientations of the procerebrum of $I$. bilineata and L. marginatus are those shown in Fig. 3C and Fig. 3D, respectively. Fractional changes in fluorescence intensity $(\Delta F / F)$ are coded as pseudo-colours; scale on right of picture. Each image was obtained by accumulating the optical signal for $38.4 \mathrm{~ms}$. Numbers in each image indicate frame number.

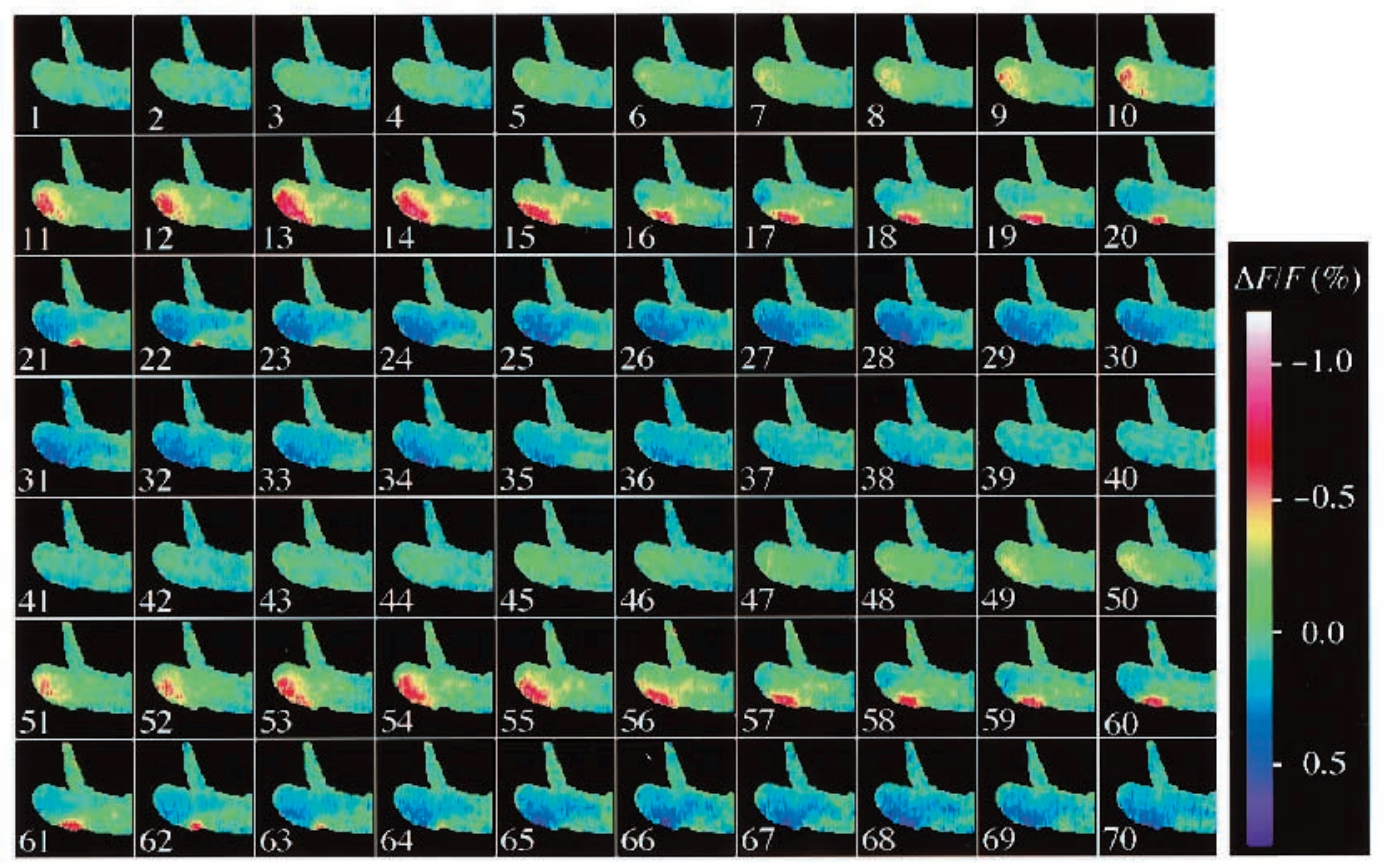

correlation between the size of the rapid component and its following peak interval. Significant correlations $(P<0.001)$ were detected in seven preparations.

\section{Discussion}

The procerebra of Incilaria bilineata and Limax marginatus were investigated using the lipophilic tracer dyes DiI and DiO
(Figs 1-3). In I. bilineata, the arrangement of the cell mass, the terminal mass and the internal mass, as well as the connection between the superior tentacle nerve and the procerebrum, were similar to those of the snails Helix pomatia and Helix lucorum rather than those of the slugs Limax maximus and Limax cinerea-niger (Zs-Nagy and Sakharov, 1970). The arrangement in L. marginatus was almost the same as that in L. maximus. In both I. bilineata and L. marginatus, 
A
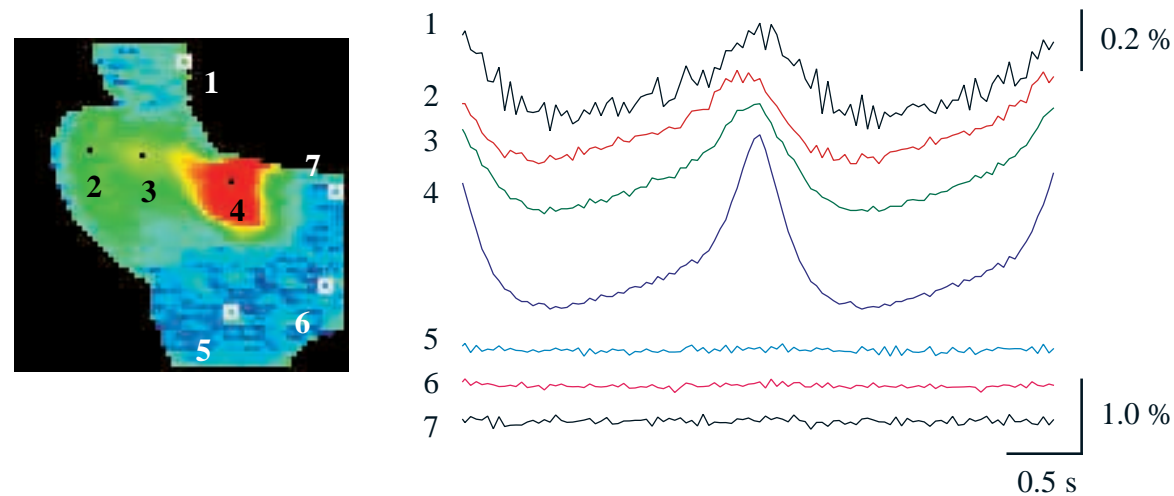

Fig. 7. The change in fluorescence intensity during one oscillatory cycle. To increase the signal-to-noise ratio, 17 cycles of oscillation in I. bilineata (A) and 23 cycles of oscillation in L. marginatus (B) were averaged after matching their depolarization peaks. Fluorescence intensity was collected from one pixel (2, cell mass; 3 , terminal mass; 4, internal mass) or $5 \times 5$ pixels (1, tentacle nerve; 5, 6, 7, regions outside the procerebrum). The upper and lower vertical scale bars represent the fractional changes in the fluorescence intensity $(-\Delta F / F)$ of the tentacle nerve and the other regions, respectively.

B

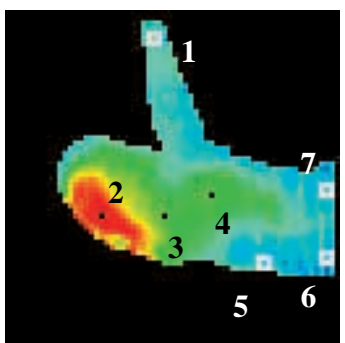

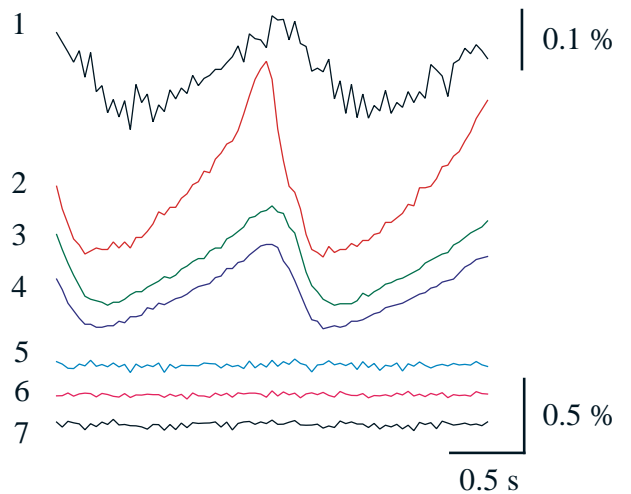

a large number of the fibres of the superior tentacle nerves projected to the terminal mass and terminated there, not entering the internal mass.

Oscillations of the local field potential of the procerebrum of $L$. marginatus were almost the same as those of $L$. maximus (Gelperin and Tank, 1990), while those in I. bilineata differed in three aspects (Figs 4, 5). First, the local field potential in $I$. bilineata oscillated mainly in the internal mass and exhibited only weak oscillations in the cell mass, while in L. marginatus both the somatic and the neurite regions showed clear local field potential oscillations. Second, the polarity of the peak of the local field potential recorded in the somatic and neurite regions was positive in the internal mass of I. bilineata and negative in the cell mass, while in L. marginatus the two peaks showed the opposite polarity. A change in the local field potential of the procerebrum of L. maximus is primarily the result of synchronous inhibitory postsynaptic currents in the procerebral neurones in the somatic region (Gelperin et al. 1993), which results in a positive peak (i.e. outward current source) in the cell mass. Therefore, the positive peak recorded in the internal mass of I. bilineata suggests the presence of synchronous inhibitory postsynaptic currents in the neurite
Fig. 8. Distribution of the halftime of the depolarization peak in the procerebrum of $I$. bilineata (A) and L. marginatus (B). The half-time of each pixel is coded as pseudo-colours and overlaid on the monochromic fluorescence image of the hemisphere of the cerebral ganglion stained with di4-ANEPPS. The key is given on the right. CM, cell mass; TM, terminal mass; IM, internal mass.
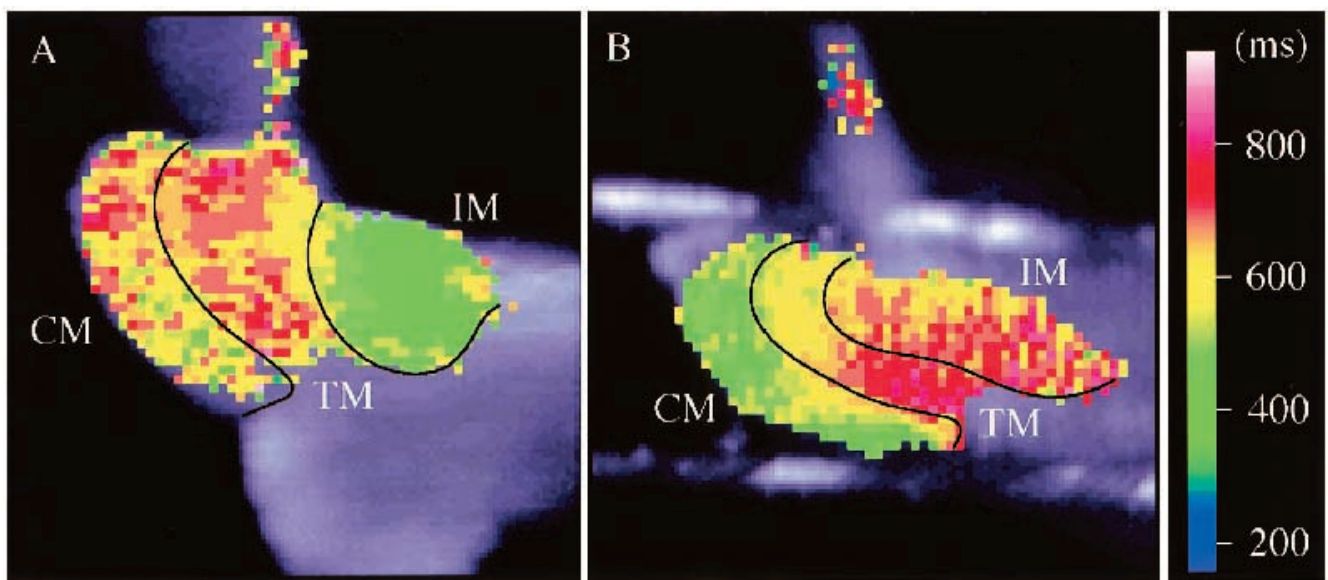
A

$\mathrm{TN}$

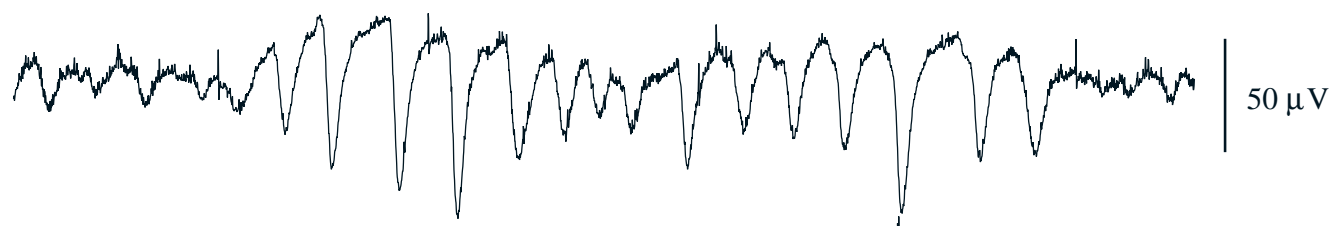

IM

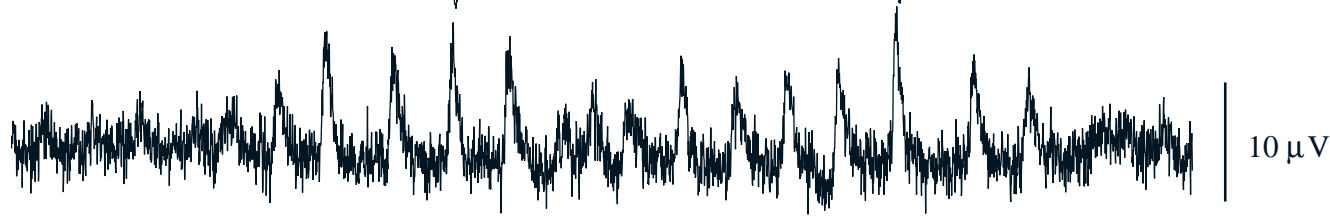

Fig. 9. Oscillations in the field potential of the tentacle nerve in synchrony with those of the procerebrum of I. bilineata (A) and L. marginatus (B). Oscillation of the tentacle nerve was recorded extracellularly from its cut end using a glass suction electrode. The tentacle nerve was cut in the middle. TN, tentacle nerve; IM, internal mass; CM, cell mass.
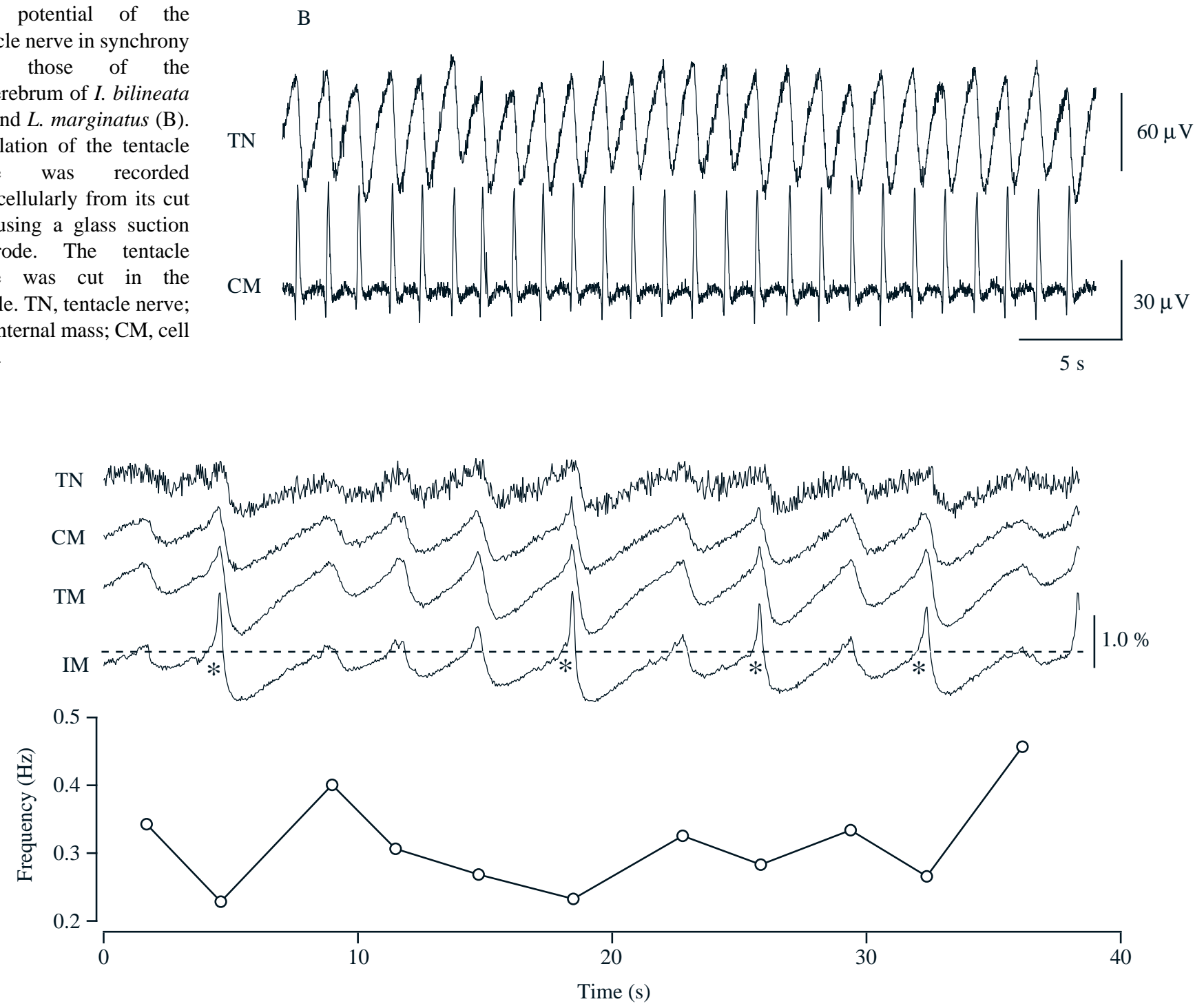

Fig. 10. Fluctuation of the amplitude and frequency of oscillation in the internal mass of I. bilineata. TN, tentacle nerve; CM, cell mass; TM, terminal mass; IM, internal mass. Fluorescence intensity was collected from $5 \times 5$ pixels located on each region to increase the signalto-noise ratio. The vertical scale bar represents the fractional change in the fluorescence intensity $(-\Delta F / F)$. Frequency was defined as the inverse of the interval between successive peaks. Asterisks indicate the large depolarization peaks which were followed by intense hyperpolarization, resulting in a decrease in frequency. The broken line indicates the border between the slow component and the rapid component. 
Fig. 11. Correlation between the size of the rapid component and the peak interval in I. bilineata. A and B are from different preparations. The abscissa shows the total fractional change in fluorescence intensity above the threshold, determined as a border-line between the slow and rapid components (see the broken line in Fig. 10), and the ordinate shows the following peak interval. Fluorescence intensity was collected from $5 \times 5$ pixels located on the internal mass. Linear regression yielded the following significant relationships: (A) $y=0.295 x+2.146$ ( $r=0.966, N=11, P<0.001)$; (B) $y=0.052 x+2.816(r=0.904$, $N=12, P<0.001)$.
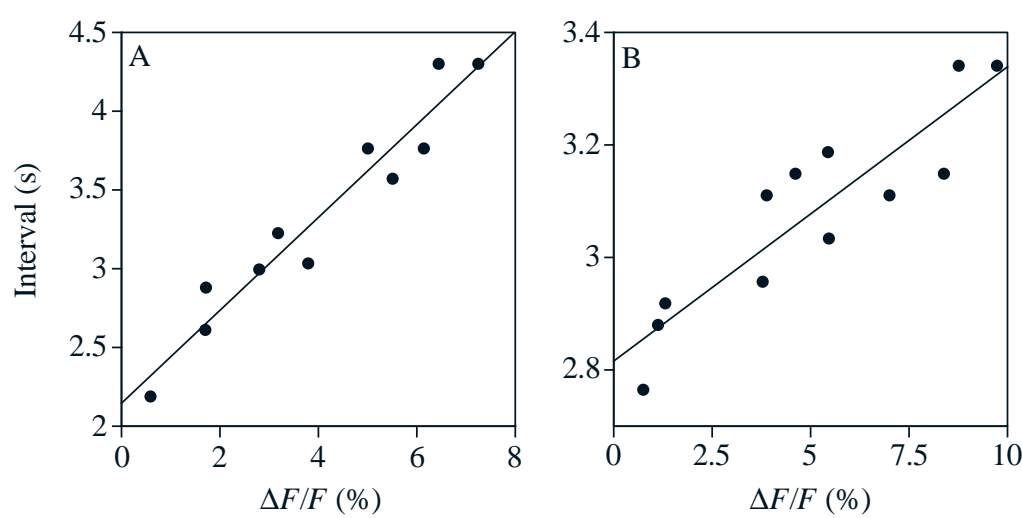

region. Third, in the in vitro study reported here, the repetitive activity of $I$. bilineata was less regular than that of $L$. marginatus with respect to amplitude and frequency. Although it is difficult to comment on the oscillatory activity of procerebral neurones in vivo, a recent study using a fine wire electrode implanted in the procerebrum of freely moving $L$. maximus (Gelperin et al. 1996) suggests that spontaneous oscillations in the intact animal are less regular than those in the isolated cerebral ganglion. If the irregularity of the oscillations in I. bilineata is representative of its properties in vivo, the oscillatory features of both species may be more similar in vivo than they appear to be in vitro.

Optical measurements revealed that differences in the oscillations of the local field potential between I. bilineata and L. marginatus are due to differences in the location of oscillatory activity in the procerebral interneurones; in $I$. bilineata, the internal mass shows the greatest oscillatory amplitude, while in L. marginatus, it is the cell mass (Figs 6, 7). Although the origin of the oscillations differs, the coherent activity of the procerebral interneurones, with a phase gradient along the distal-proximal axis, is common to both species. Since the superior tentacle nerve, which conveys olfactory information, projects to the terminal mass, oscillation of the membrane potential in the adjacent region might affect the transmission of olfactory information to the procerebral interneurone in the terminal mass. It has been reported that the amplitude of the procerebral field potential evoked by electrical stimulation of the superior tentacle nerve depends on the timing of the electrical stimulus relative to the phase of oscillation (Gelperin and Tank, 1990). One possible mechanism for this phase-dependent phenomenon is modulation of activity in the tentacle nerve in synchrony with the procerebral oscillation. This hypothesis is supported by the results shown in Figs 7 and 9. The membrane potentials of the axons constituting the superior tentacle nerve oscillate in synchrony with the oscillations of the procerebral neurones. This suggests that efferent modulatory synapses may exist in the terminal mass and transmit information on the macroscopic state of the procerebral neural circuit to the superior tentacle nerve. However, because the superior tentacle nerve undoubtedly contains both afferent and efferent fibres, future experiments should investigate whether the procerebral oscillations affect the neural activities of the afferent fibres, the efferent fibres or both. The relationship between sensory neurones and a higher rhythm-generating neural network has been observed in buccal feeding motor systems, where the duration of the afferent spike of a radular mechanosensory neurone is modulated centrally and depends on the phase of the buccal motor rhythm (Kawahara et al. 1994) which, in turn, is activated by excitation of the mechanosensory neurone (Kawahara et al. 1995).

An analysis of optical signals revealed that the waveform in the internal mass of $I$. bilineata and in the cell mass of $L$. marginatus is distinct from, and sharper than, that in other regions (Figs 7, 8, 10). We interpret the waveform as bursting activities superimposed on the subthreshold oscillations of membrane potential which are dominant in other regions. In $L$. maximus, rapid depolarization measured optically has been assigned to the signal coming from the bursting neurones, while slow depolarization is generated by the non-bursting neurones (Delaney et al. 1994; Kleinfeld et al. 1994), although the contribution of the signal coming from the neurite region just under the cell layer is not known. In this study, however, part of the difference in the optical signal seemed to relate to regional differences in the membrane properties of the procerebral interneurones. Because the projection pattern of the procerebral neurones preserves an ordered arrangement of cell bodies along the distal-proximal axis (Fig. 3), optical signals from cell bodies located in the cell mass should resemble those from the internal mass if the membrane properties of the procerebral neurones are the same over the whole length of the cell. It is possible that, in L. marginatus, bursting neurones may not have neurites in the internal mass as well as in the terminal mass, while the non-bursting neurones definitely have neurites in both. However, this is not applicable to I. bilineata, which has the rapid component in the internal mass. Because input and output pathways project to the terminal mass (Chase and Tolloczko, 1989; Gelperin et al. 1993) and the superior tentacle nerve oscillates with the procerebrum interneurones (Figs 7, 9), slow oscillations in the terminal mass may be the product of interactions between an intrinsic membrane property of procerebral interneurones and input and output pathways. Consequently, the membrane potentials in the cell mass of $I$. bilineata and in the internal mass of L. marginatus may be 
strongly affected by the membrane potential in the terminal mass and, therefore, resemble it (Figs 7, 8).

The data from I. bilineata suggest a recurrent relationship between the slow and the rapid components. In some preparations of $I$. bilineata, which showed irregular oscillations, the degree of hyperpolarization after the depolarization peak depended on the size of the rapid component, and the time interval until the next peak depended on the degree of hyperpolarization (Figs 10,11). We propose that the slowly depolarizing component activates the rapid component, which in turn inhibits or resets the slow component, depending on the degree of activation of the rapid component. In L. marginatus, we found that the frequency of oscillation correlates well with the cycle-averaged field potential recorded optically in the terminal mass, when stimulated with pleasant or unpleasant odours (Toda et al. 1996), which also supports the hypothesis that the degree of depolarization (or hyperpolarization) in the terminal mass, which largely showed the slow component, affects the frequency of oscillation. This kind of recurrent inhibition has been observed in the vertebrate olfactory bulb (Shepherd, 1972) and may be the origin of the oscillatory pattern (Freeman, 1987; Eeckman and Freeman, 1990). The olfactory centre of the terrestrial slug may perform an operation similar to that of mammals, although the underlying neural structures involved are likely to be different.

This work was supported by Grants-in-Aid for Scientific Research from the Ministry of Education, Science, Sports and Culture, Japan (nos. 08680714, 07557143 and 07279105) and in part by a Grant from the Program for Promotion of Basic Research Activities for Innovative Biosciences.

\section{References}

Chase, R. (1985). Responses to odors mapped in snail tentacles and brain by $\left[{ }^{14} \mathrm{C}\right]$-2-deoxyglucose autoradiography. J. Neurosci. 5, 2930-2939.

Chase, R. And TolloczKo, B. (1989). Interganglionic dendrites constitute an output pathway from the procerebrum of the snail Achatina fulica. J. comp. Neurol. 283, 143-152.

CoOKe, I. R. C. AND GelPerin, A. (1988). Distribution of FMRFamide-like immunoreactivity in the nervous system of the slug Limax maximus. Cell Tissue Res. 253, 69-76.

Croll, R. P. AND Chase, R. (1980). Plasticity of olfactory orientation to foods in the snail Achatina fulica. J. comp. Physiol. A 136, 267-277.

Delaney, K. R., Gelperin, A., Fee, M. S., Flores, J. A., Geravais,
R., TANK, D. W. AND Kleinfeld, D. (1994). Waves and stimulusmodulated dynamics in an oscillating olfactory network. Proc. natn. Acad. Sci. U.S.A. 91, 669-673.

Eeckman, F. H. and Freeman, W. J. (1990). Correlations between unit firing and EEG in the rat olfactory system. Brain Res. 528, 238-244.

FreEMAN, W. J. (1987). Simulation of chaotic EEG patterns with a dynamic model of the olfactory system. Biol. Cybernetics 56, 139-150.

Gelperin, A., Kleinfeld, D., DenK, W. And CoOKe, I. R. C. (1996). Oscillations and gaseous oxides in invertebrate olfaction. $J$. Neurobiol. 30, 110-122.

Gelperin, A., Rhines, L. D., Flores, J. And Tank, D. W. (1993). Coherent network oscillations by olfactory interneurons: modulation by endogenous amines. J. Neurophysiol. 69, 1930-1939.

GelPERIN, A. AND TANK, D. W. (1990). Odour-modulated collective network oscillations of olfactory interneurons in a terrestrial mollusc. Nature 345, 437-440.

Kawahara, S., Yano, M. And Shimizu, H. (1994). Radular mechanosensory neuron in the buccal ganglia of the terrestrial slug, Incilaria fruhstorferi. J. comp. Physiol. A 174, 111-120.

KaWAHARA, S., Yano, M. AND ShimizU, H. (1995). Modulation of the feeding system by a radular mechanosensory neuron in the terrestrial slug, Incilaria fruhstorferi. J. comp. Physiol. A 176, 193-203.

Kimura, T., Suzuki, B., Yamada, A., Sekiguchi, T. And Mizukami, A. (1993). Response of oscillatory field potential to some conditioned odors in slug's brain. Zool. Sci. 9, 1241.

Kleinfeld, D., Delaney, K. R., Fee, M. S., Flores, J. A., Tank, D. W. AND GELPERIN, A. (1994). Dynamics of propagating waves in the olfactory network of a terrestrial mollusc: an electrical and optical study. J. Neurophysiol. 72, 1402-1419.

SAHLEY, C., Rudy, J. W. AND GelPERIN, A. (1981). An analysis of associative learning in a terrestrial mollusc: Higher-order conditioning, blocking and a transient US pre-exposure effect. $J$. comp. Physiol. A 144, 1-8.

SHEPHERD, G. M. (1972). Synaptic organization of the mammalian olfactory bulb. Physiol. Rev. 52, 864-917.

TEYKe, T. (1995). Food-attraction conditioning in the Roman snail, Helix pomatia. J. comp. Physiol. A 177, 409-414.

Toda, S., Kimura, T., Kawahara, S., Sekiguchi, T. and Kirino, Y. (1996). Optical study of central olfactory processing in the terrestrial slug. Neurosci. Res. (Suppl.) 20, S223.

Yamada, A., Sekiguchi, T., Suzuki, H. And Mizukami, A. (1992). Behavioral analysis of internal memory states using coolinginduced retrograde amnesia in Limax flavus. J. Neurosci. 12, 729-735.

Zs-NAGy, I. AND SAKharov, D. A. (1970). The fine structure of the procerebrum of pulmonate molluscs, Helix and Limax. Tissue \& Cell 2, 399-411. 